\title{
DISPOSITIVOS MÓVILES EN LA EDUCACIÓN: TENDENCIAS E IMPACTO PARA LA INNOVACIÓN
}

\author{
Yois Smith Pascuas-Rengifo ${ }^{1}$, John Arley Garcia-Quintero ${ }^{2}$, Martin Alonso Mercado-Varela ${ }^{3}$ \\ 1 Doctora en Educación y Cultura Ambiental, docente de aula Secretaría de Educación Municipal Florencia \\ Caquetá - Colombia. \\ 2 Magister en E-Learning y Redes Sociales, docente ocasional tiempo completo de la Facultad de ingeniería \\ de la Universidad de la Amazonia - Colombia. \\ ${ }^{3}$ Doctor en Ciencias Educativas, docente investigador del Instituto Tecnológico de Sonora - México.
}

Email: y.pascuas@udla.edu.co,j.garcia@udla.edu.co,martin.mercado@itson.edu.mx

\section{RESUMEN}

La innovación es fundamental para el desarrollo de nuevos productos que contribuyan a la generación de valor en la sociedad. Dentro de la educación, la integración de tecnologías de la información y las comunicaciones ha posibilitado la innovación de los procesos de enseñanza-aprendizaje, como es el caso de la utilización de dispositivos móviles (M-Learning) dentro y fuera del salón de clase. En la presente investigación se analiza producción académica sobre la utilización de dispositivos móviles con fines educativos, privilegiando el análisis de las tendencias e impacto. Los principales hallazgos de esta investigación evidencian que la adaptación del M-Learning debe ser una prioridad dado que es la base para el desarrollo de otras estrategias como la realidad aumentada, realidad virtual, realidad mixta, gamificación, laboratorios virtuales y remotos, y la integración de la inteligencia artificial en pro de fortalecer los desafíos del sistema educativo.

Palabras clave: innovación educativa; dispositivos móviles; aprendizaje móvil; tecnologías de la información y la comunicación; tendencias educativas.

Recibido: 25 de Febrero de 2020. Aceptado: 12 de Mayo de 2020.

Received: February 25, 2020. Accepted: May 12, 2020.

\section{MOBILE DEVICES IN EDUCATION: TRENDS AND IMPACT FOR INNOVATION}

\begin{abstract}
Innovation is essential for the development of new products that contribute to the generation of value in society. Within education, the integration of information and communication technologies has enabled the innovation of teaching-learning processes, as is the case of the use of mobile devices (M-Learning) inside and outside the classroom. This research analyzes academic production on the use of mobile devices for educational purposes, favoring the analysis of trends and impact. The main findings of this research show that the adaptation of M-Learning must be a priority since it is the basis for the development of other strategies such as augmented reality, virtual reality, mixed reality, gamification, virtual and remote laboratories, and integration of artificial intelligence in order to strengthen the challenges of the educational system.
\end{abstract}

Keywords: educational innovation; mobile devices; mobile learning; technology of the information and communication; educational trends.

Cómo citar este artículo: Y. Pascuas, J. Garcia, M. Mercado. "Dispositivos móviles en la educación: tendencias e impacto para la innovación", Revista Politécnica, vol.16, no.31 pp.97-109, 2020. DOI: 10.33571/rpolitec.v16n31a8 


\section{INTRODUCCIÓN}

La innovación es una prioridad de los gobiernos, el valor de las creaciones intelectuales está por encima de las materias primas que pueda poseer un país [1]. La educación es uno de los campos que están experimentando importantes innovaciones, debido a la necesidad de romper paradigmas obsoletos que no convergen con las realidades de la sociedad y la desmasificación de las actuales generaciones, las cuales se encuentran atiborradas de medios de información y comunicación, que eliminan las barreras de inserción a la educación, al punto de considerarse que la academia ha perdido el monopolio del conocimiento.

Las TIC ofrecen importantes oportunidades a la sociedad, pues la inmediatez, la ubicuidad y la reducción de la necesidad de traslado son las características más relevantes de estas herramientas. Además, hacen parte de la vida cotidiana de las personas, su inmersión en el campo educativo es una realidad que ha dado paso al descubrimiento estilos de aprendizaje dados por ejemplo por los dispositivos móviles o lo denominado Mobile Learning (por sus siglas en inglés $M$-Learning). Estrategia que permite llevar el proceso educativo más allá del aula de clases, transformando el papel de los actuales docentes, donde el acceso a la información se ha democratizado a través contenidos y plataformas actualizadas. Asistir a los centros educativos es una actividad que no se limita a la adquisición de información, sino a enriquecer el pensamiento a través del desarrollo de competencias actitudinales, ciudadanas y disciplinares.

El M-Learning es una estrategia que involucra el uso de dispositivos móviles, especialmente celulares y tabletas, en el proceso de aprendizaje, el cual requiere del desarrollo tanto de software como de hardware, permite la interacción constante entre pares y docentes, haciendo del proceso de aprendizaje un espacio de intercambio de ideas y articulación de agentes del ecosistema educativo $[2,3]$.

El siguiente artículo contiene una revisión sistemática de literatura sobre las tendencias e impacto del el M-Learning y cuyo objetivo es pretender responder a interrogantes planteados. Asimismo, se encuentra conformado por tres secciones: en primer lugar, se hace la conceptualización; en segundo lugar, se realiza una revisión de las experiencias relacionadas y finalmente se hace un análisis del impacto y las tendencias que presenta el M-Learning como estrategia dentro de las innovaciones educativas que integran las TIC.

\section{MATERIALES Y METODO}

La revisión de literatura es un proceso de gran apoyo para el conocimiento científico, ya que los hallazgos y contribuciones que se generan luego de la búsqueda, ordenamiento y análisis crítico de los documentos seleccionados, generan un conocimiento actualizado sobre el tema de interés, además de identificar tendencias de investigación y soporte para nuevas $[4,5]$.

De esta manera, el presente articulo presenta la selección de estudios primarios sobre prácticas y experiencias de M-Learning, se realizaron consultas en diferentes bases de datos y motores de búsqueda, fuentes válidas y confiables de información académica y científica como: Scopus, Springer, Science Direct, Association for Computing Machinery y Scielo. El criterio de búsqueda y selección de documentos estuvo relacionado bajo las palabras claves $M$-Learning, Mobile Learning, tendencias del M-Learning, e impacto del aprendizaje móvil de la producción académica publicada entre el 2014-2018, con el objetivo principal de responder los siguientes interrogantes planteados:

1) ¿Cuáles son las principales tendencias en la utilización del M-Learning?

2) ¿Cuál ha sido el impacto sobre los procesos de enseñanza-aprendizaje del M-Learning?

El proceso de búsqueda incluyó la identificación de artículos científicos, capítulos de libros, libros especializados y memorias de evento, que presentarán relación con experiencias relacionadas con las palabras claves y con las preguntas orientadoras. De los estudios encontrados se excluyeron los documentos que no pertenecían a revistas indexadas y de reconocimiento académico y científico o que no hayan tenido un proceso de evaluación y control de calidad realizada por pares académicos. 


\section{RESULTADOS}

\subsection{Tecnología móvil para innovar}

El término TIC se ha masificado de manera considerable en muchos campos, ha sido el responsable en gran medida de lo que se denomina la sociedad del conocimiento; y es gracias a la innovación que estas herramientas permiten la recolección, almacenamiento, tratamiento, difusión y transmisión de información que genera impacto en la sociedad [6].

La innovación se podría denominar como la generación de una mejor versión de procesos y productos a través del uso de conocimiento antiguo y/o nuevo, con el fin de obtener beneficios generalmente económicos [7]. Innovar es un proceso que requiere de entrenamiento constante, pensamiento crítico y un alto sentido de premura para determinar el momento de desaprender, aprender o reaprender; esto acompañado de una rica interacción con equipos de trabajo [8]. La colaboración de estos equipos es fundamental en cualquier proceso de innovación, pues, el tránsito de ideas no debe verse limitado por su origen, sino por su capacidad de impacto, lo que ha permitido que pares e incluso competidores colaboren para compartir riesgos [9].

Aunque los términos TIC e innovación se han asociado generalmente a la ingeniería computacional, es de resaltar el impacto que han tenido en el campo educativo. La innovación educativa es la introducción de cambios en los procesos formativos y de aprendizaje [10], los cuales deben ser sostenibles, replicables, adaptables, eficientes y eficaces. Aunque el proceso requiera la introducción de tecnología, ésta no debe ser la condición preponderante, ya que están para facilitar y acondicionar la implementación de enfoques pedagógicos. La innovación educativa contribuye a la integración socioeducativa de los estudiantes [11], la cual debe tener como características fundamentales la equidad, colaboración y participación de todos los agentes educativos.

Por su parte se plantea que la innovación educativa apoya el aprendizaje, la profesionalización, la cobertura, la graduación, ya que llena de confianza a los estudiantes y estimula la necesidad de avanzar [12]. Además, reinventa la práctica docente, lo que conduce a mejorar significativamente la autoestima y sentido de pertenencia por la institución. Sin embargo, ello implica el cambio en patrones culturales [13].

En adición, la implementación de las TIC en el proceso educativo contribuye a que los estudiantes tengan mayor deseo de aprendizaje y adquirir autonomía, ya que es un medio flexible, dinámico, y que permite estar en contacto con equipos de trabajo [14]. Sin embargo, las perspectivas no son las únicas que se modifican, pues, patrones de comportamiento también se ven alterados al introducir innovaciones en la educación. Por otro lado, estos procesos de transformación educativa permiten una reorientación en el papel del aprendizaje, pues gracias a la tecnología se pueden organizar ecosistemas de trabajo que resuelvan problemas auténticos del mundo real, para ello, un profesor podría utilizar una lista de competencias individuales como: la resolución creativa de problemas, el pensamiento sistémico, la orientación a objetivos; y grupales como: el trabajo en equipo y redes; donde el estudiante es capaz de identificar su habilidades y debilidades, trabajar en pro de ello y contextualizarse con el mercado laboral $[15,16,17]$.

Por su parte, se afirma que las TIC presentan la información de manera fácil, práctica, divertida y dinámica, facilitando el contacto con muchas personas y propiciando una educación global y a distancia, pero al ser abundante e inmediata puede que se convierta en un problema, pues lo estudiantes limitarían su pensamiento y podrían recurrir a prácticas como el plagio, es allí donde el papel del docente es preponderante al estimular prácticas éticas que contribuyan al cuidado y enriquecimiento del conocimiento científico [18].

La integración de las TIC en entornos educativos ha permitido la constante innovación en los procesos de formación, tanto así, que se han generado nuevos modelos de educación no presenciales como el $M$-Learning, el cual se presenta como una modalidad educativa alternativa a la tradicional, en donde el principal elementos mediador tecnológico es el celular, la tableta o cualquier dispositivo electrónico móvil, que permita la interacción con el contenido, con acceso desde cualquier momento y lugar, además de la constante obtención de información debido a la intercomunicación entre el docente y los estudiantes, facilitando de esta manera la adquisición de habilidades y/o destrezas [19]. 
Es así como se denomina el M-Learning como "la integración del e-learning con los dispositivos digitales portátiles como teléfonos móviles, agendas electrónicas, tabletas, lectores de e-book, entre otros. Los dispositivos digitales portátiles permiten a las personas tener experiencias formativas en cualquier momento y lugar (ubicuidad).", característica esencial del $M$ Learning. De este modo, el avance del proceso de formación en línea ha permitido la creación de ambientes completos de formación como los LMS (Learning Management Systems); permitiendo a las instituciones educativas de diferentes niveles, la oferta de sus servicios educativos a través de Internet aplicando la comunicación sincrónica y asincrónica [20, 21].

\subsection{Tendencias y aplicabilidad de M-learning}

Los dispositivos móviles se han convertido en una importante herramienta en la vida cotidiana, tanto en el plano laboral, académico, familiar, social y recreativo. Aunque no existe una definición exacta, se entiende que son todos aquellos dispositivos electrónicos que son de fácil acceso, uso y portabilidad; entre los más destacados están: los reproductores de música, grabadoras, GPS, lectores de libros, tableta y smartphone. Siendo estos dos últimos los de mayor uso, pues tienen la capacidad de ejecutar tareas de los anteriores [22].

El incremento del uso de dispositivos móviles en la educación ha dado origen al concepto de Mobile Learning. La adquisición de conocimiento, habilidad y actitudes a través del uso de dispositivos móviles [23], los cuales deben ser evaluados con criterios de adaptabilidad, rendimiento y eficiencia por las instituciones, de acuerdo con las necesidades particulares que presente el ecosistema de aprendizaje [24]. Las características más relevantes del aprendizaje móvil es la posibilidad de revisar ilimitadamente los contenidos; entenderlos de una mejor manera, ya que se encuentran organizados y estructurados [25].

Los beneficios no son únicamente para los estudiantes, pues los docentes fortalecen el proceso de enseñanza al tener mayor participación de estudiantes en las actividades [26]. Los dispositivos móviles al beneficiar a ambas partes en el proceso educativo, contribuye a satisfacer las necesidades de los estudiantes, impartir clases con eficiencia, dinamizar y mejorar el aprendizaje, romper paradigmas de la educación tradicional y llevar el aprendizaje más allá de la institución [27].

No obstante, los dispositivos móviles en el aula de clase pueden traer consecuencias negativas como la distracción, la falta de habilidades prácticas y la reducción de la interacción cara a cara; pues al ser multitareas no necesitan tener una aplicación exclusiva con contenidos académicos, es decir, se convierten en una herramienta genérica de uso recurrente [28]. Al respecto cuestionan que aunque se usen aplicaciones con destinación educativa, muchas de ellas no poseen pruebas de usabilidad ni revisión de expertos, debido a la falta de directrices y metodologías de evaluación [29].

El uso de los dispositivos móviles en la educación se masificó considerablemente con los desarrollos y prueba piloto que realizó Nicholar Negroponte con tabletas digitales en países subdesarrollados. Demostraron que dotando de tabletas a estudiantes sin ninguna directriz, éstos se muestran más motivados, pues los dispositivos adquieren el calificativo de herramienta versátil al permitir usar la cámara como fotocopiadora, reproducir contenidos y realizar consultas en la web, además de permitir al docente crear nuevas actividades [30].

A través del uso de una aplicación móvil (app) en el estudio del ciclo de vida de los árboles [31], demostraron que los estudiantes se motivan a participar en el aprendizaje en un entorno natural, desarrollan prácticas para abordar problemas y toma de decisiones en tiempo real. Por otra parte se demostró que a través de una app de gestos se puede contribuir significativamente al fortalecimiento de habilidades cognitivas de personas con necesidades educativas especiales [37]. Las tabletas se usan mayoritariamente en consumo, creación y uso compartido de contenidos, además, se destaca que los estudiantes incrementan su interacción y el trabajo colaborativo $[32,33]$.

El desarrollo tecnológico de la tableta ha sido alto, dejando una frontera de diferenciación muy delgada con los smartphones, pues el tamaño de pantalla a veces es la única discrepancia, lo cual genera cambios en la experiencia educativa. En relación a esto se analizó la experiencia de estudiantes con smartphones de 3,5" y tabletas de 7" y 10", encontrando que una pantalla de 3,5" genera desventaja frente a los demás dispositivos, pero a 
partir de 5" la experiencia no sufre afectaciones significativas [33].

En el proceso educativo una de las materias de mayor dificultad para los estudiantes son las matemáticas, frente a esto, aprovechando la precisión de los sensores de los dispositivos móviles [34], se integró la aplicación GeoGebra a la clase de física cuántica, logrando establecer un laboratorio móvil. También se identificó una experiencia relacionada con ecuaciones cuadráticas y conceptos de materiales $[35,36]$ experiencias que relacionan un alto porcentaje de aceptación.

En el sistema educativo tradicional, la evaluación causa estrés y ansiedad entre los estudiantes, al respecto se plantea que usando la aplicación Kahoot [38] para la elaboración y aplicación de pruebas de conocimiento interactivas al final de cada clase, los estudiantes logran identificar sus debilidades y se motivan a mejorar los resultados. Sin embargo, diversas experiencias coinciden en que la entrega de contenidos a través de dispositivos móviles no es suficiente para elevar el rendimiento académico de los estudiantes, pero proporciona elementos que estimulan la permanencia en el proceso $[39,40]$. Por otro lado, a través de la app Ruzo [41] monitorearon el progreso de los estudiantes de un curso de metodología de la investigación, demostrando mayor participación y motivación, lo cual no se había logrado con métodos flexibles de calificación.

A su vez se diseñó una app de aprendizaje del idioma inglés [42], en la cual los estudiantes no se limitaban únicamente a consumir contenidos, se convierten en creadores y evaluadores, permitiendo mayor interacción y unificación de intereses colectivos, pues, aunque las tareas se realizan de manera individual, estas terminan afectando a todo el grupo, y dan pautas a los docentes para la creación de contenidos básicos. De otra parte, se hizo seguimiento al estado cognoscitivo de los estudiantes midiendo el ritmo cardiaco mediante la aplicación AttentiveLearner, lo cual permitió evaluar la calidad de los cursos masivos abiertos en línea (MOOC) que estaban desarrollando los estudiantes [43].

\subsection{Impacto del M-learning en la formación}

Diversas experiencias del $M$-Learning se basan en la incorporación de aplicaciones disponibles en las tiendas; no obstante, estas son limitadas al momento de adaptarse a las necesidades de cada ecosistema. Ante ello se han diseñado la plataforma JEM iNVENTOR [44] que permite al docente crear aplicaciones de acuerdo con los requerimientos específicos del curso, para ello cuenta con tres niveles de apropiación, pues no todos tienen los conocimientos técnicos en informática. En este sentido, se enfatizan sobre la importancia de la adaptación de los recursos como fundamental a la hora de implementar el $M$ Learning [45], pues de ello depende el éxito del proceso, el cual requiere de tiempo para realizar ajustes y ver la evolución de los estudiantes. Dejando planteado que el objetivo principal del $M$ Learning es crear escenarios de participación masiva, eliminando fronteras donde las limitaciones físicas y cognitivas no sean un impedimento [46].

Como se evidenció en las anteriores experiencias, el uso del M-Learning es relativamente joven comparado con otras innovaciones educativas que integran TIC; y su desarrollo es directamente proporcional al de las conexiones inalámbricas y propiedad de los dispositivos. En este sentido, docentes y alumnos se muestran interesados en adoptar este tipo de estrategias, evidenciándose disminución de la resistencia por parte de los docentes [47], aunque existen barreras de entrada como la cobertura, seguridad y competencias digitales. Sin embargo, se demuestra que esta generación no es la más beneficiada con el $M$ Learning [48], pues los estudiantes adultos por cuestiones de tiempo y trabajo son los más propensos a adoptarla adecuadamente.

La democratización de los dispositivos móviles ha sido una preocupación para países en vía de desarrollo, pues es un medio indispensable para la dinámica económica, social y educativa. Al respecto Colombia dio un importante paso con la Ley 1819 de 2016 en la cual elimina el cobro de impuestos a algunos teléfonos inteligentes. Los cuales brindan las mismas posibilidades de acceso a información y aplicaciones de redes sociales. Justamente éstas se han convertido en un medio informal para llevar la clase más allá de las aulas; y son consideradas eficaces para mejorar la participación y cerrar la brecha entre las 
instituciones y los nativos digitales [49]. En donde se pueda, por ejemplo, personalizar las redes sociales a través de notificaciones que estimulen el aprendizaje y la administración del tiempo [50].

Otra tendencia del M-Learning es incursionar en la realidad aumentada y virtual, pues de acuerdo con el éxito de Pokemon Go [51] es una importante evidencia del poder que tiene esta tecnología para motivar la participación de los estudiantes, pero su proceso será más lento, pues, aunque en la actualidad existe un cumulo de posibilidades a través del software libre, el costo y desarrollo de hardware es un limitante. No obstante, se plantean evitar interacciones artificiales y mejor integrar los dispositivos a situaciones reales [52], pues saca al estudiante de su zona de confort, mejora la competencia de resolución de problemas y asimilación del fracaso.

Hasta el momento se han mencionado los beneficios del $M$-Learning, pero es importante tener en cuenta que esta estrategia tiene retos por enfrentar. Las adicciones del comportamiento [53], la nomofobia es una de las más complejas de entender y tratar, pues no es fácil determinar en qué momento el dispositivo móvil deja de ser una herramienta de uso cotidiano y se convierte en un instrumento adictivo, generando un círculo vicioso de procrastinación. Adicionalmente, se considera que existe suficiente evidencia para demostrar que los dispositivos móviles en clase están íntimamente ligados con el cyberbullying, el sexting, el fraude en evaluaciones y las interrupciones en clase $[54,55]$. Ante este panorama Colombia propone a través del proyecto de Ley 099 de 2018 la prohibición de celulares en centros educativos. Sin embargo, dadas las condiciones del sistema educativo, los dispositivos móviles son necesarios a pesar de los riesgos, ya que genera motivación en los estudiantes y abre un abanico de oportunidades para trabajar con ellos [56].

En este orden de ideas, existen diferentes posturas frente al $M$-Learning, existen debilidades frente a su implementación como: conectividad a la red debe ser constante, las baterías de los dispositivos no duran mucho tiempo, existen aplicaciones complejas para administrar, y el bajo nivel de competencias TIC de los docentes [57]. Por otro lado, se establece la existencia de amenazas asociadas al uso excesivo e intensivo de los móviles como el estrés, insomnio, sexting, nomofobia, entre otras [58]. Pero lo negativo con la implementación del aprendizaje móvil [59], podría desencadenar el desarrollo de tecnológico, mejorar los entornos educativos en línea, el desarrollo de metodologías emergentes, es decir, aumentar las investigaciones del aprendizaje personalizado y experiencias inmersivas por medio de la realidad aumentada y virtual, a demás de las fortalezas asociadas a la educación mediada por la tecnología móvil. En la Figura 1, se resume en una matriz DOFA (debilidades, oportunidades, fortalezas y amenazas) las diferentes posturas de la implementación del M-Learning.

\section{DEBILIDAD}

1. Conexión a internet

2. Aplicaciones rígidas almacenamiento

4. Duración reducida de las baterías

5. Débil competencia TIC de los docentes

\section{FORTALEZA}

1. Propiedad de los equipos

2. Ubicuidad

3. Mayor participación

4. Contenidos actualizados

5. Disminución de la huella ambiental
3. Limitados recursos de
OPORTUNIDAD

1. Infraestructura WIFI

2. Desarrollo de metodologías

3. Investigaciones en impacto cuantitativo

4. Realidad aumentada y virtual

5. Contenidos personalizados

\section{AMENAZA}

1. Restricción legal

2. Sexting

3. Ciberbullying

4. Nomofobia

5. Interrupciones en clase
Fig.1. Matriz DOFA del M- Learning

Es así como el modelo M-Learning presenta oportunidades para otros entornos de aprendizaje como los MOOC, pues permitiría mayor participación y alcance, sin embargo, tiene dificultades para ser competitivos frente a los ambientes de escritorio, ya que la interacción se limita en el manejo de multitareas, pantallas reducidas, almacenamiento y teclado [60]. Al respecto se propone que estos limitantes vislumbran una oportunidad de articulación del $M$ Learning con el aprendizaje electrónico (e-learning) [61], es decir, que estos modelos no deben competir entre ellos, sino buscar lo mejor de cada uno para ofrecer experiencia de aprendizaje ricas en contenidos y reducir la dependencia de las conexiones a Internet, a través del desarrollo de aplicaciones offline. En la Tabla 1, se presentan las 
características de los dos modelos presentada por [61] en donde se resaltan como aspectos positivos las flechas hacia arriba y los aspectos negativos las flechas hacia abajo.

\begin{tabular}{|c|c|c|}
\hline Características & M-Learning & E-Learning \\
\hline Red & $\begin{array}{l}\text { Inalámbrico, } \\
\text { GPRS, G3, } \\
\text { Bluetooth }\end{array}$ & $\begin{array}{l}\text { Cableado, } \\
\text { ancho } \\
\text { banda } \downarrow\end{array}$ \\
\hline Dispositivos & Smartphones $\downarrow$ & $\begin{array}{l}\text { Computadores } \\
\uparrow\end{array}$ \\
\hline $\begin{array}{ll}\text { Acceso } & \text { vía } \\
\text { Internet } & \\
\end{array}$ & Ubicuidad $\uparrow$ & $\begin{array}{l}\text { Servicio de } \\
\text { telefonía fija } \downarrow\end{array}$ \\
\hline Comunicación & $\begin{array}{l}\text { Sencillo vía } \\
\text { SMS y MMS } \uparrow\end{array}$ & Interactivo $\downarrow$ \\
\hline $\begin{array}{l}\text { Intercambio de } \\
\text { archivos }\end{array}$ & $\begin{array}{l}\text { Fácil de usar, } \\
\text { bluetooth y } \\
\text { tecnología IR } \\
\uparrow\end{array}$ & Difícil $\downarrow$ \\
\hline Almacenamiento & Ineficaz $\downarrow$ & Eficiente $\uparrow$ \\
\hline $\begin{array}{l}\text { Protección de la } \\
\text { comunicación }\end{array}$ & $\begin{array}{l}\text { Alta } \\
\text { Protección por } \\
\text { usar } \\
\text { dispositivos } \\
\text { personales } \uparrow\end{array}$ & $\begin{array}{l}\text { Baja } \\
\text { múltiples } \\
\text { usuarios en el } \\
\text { mismo } \\
\text { dispositivo } \downarrow\end{array}$ \\
\hline
\end{tabular}

Tabla 1. Características del M-Learning y $E$ Learning adaptado de [61]

Así mismo, en la Figura 2 se presenta el tipo de dispositivos móviles utilizados en los procesos de enseñanza aprendizaje en diferentes experiencias relacionadas con los dispositivos móviles [62], reflejando que el $47 \%$ de los estudios usaron dispositivos móviles tradicionales, el $4 \%$ usaron tabletas, el $11 \%$ usaron teléfonos inteligentes, $20 \%$ no limitaron los tipos de dispositivos, mientras que el $18 \%$ no usó dispositivos, lo que indica que esos estudios son en un alto porcentaje teóricas.

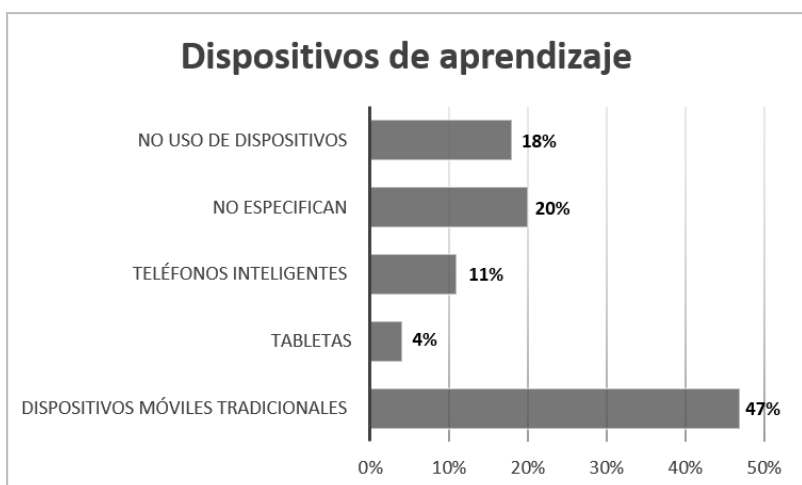

Fig.2. Dispositivos de aprendizaje adaptado de [62]
En cuanto a la Figura 3, las actividades de aprendizaje que realizaban con los dispositivos móviles, reflejan que el $22 \%$ de los estudios argumentan que los estudiantes emplearon dispositivos móviles para acceder a materiales de aprendizaje, el $7 \%$ guiaron a los estudiantes a acceder a materiales de aprendizaje y realizar evaluaciones a través de dispositivos móviles, y el $21 \%$ les brindó a los estudiantes un entorno de aprendizaje que consistía en un soporte en línea completo, lo que indicaba proporcionar a los estudiantes orientación o comentarios basados en su situación de aprendizaje. Además, el $24 \%$ de los estudios empleó dispositivos móviles para ayudar a los estudiantes a aprender en entornos virtuales y reales. Sin embargo, el $5 \%$ de los estudios no mencionó claramente los roles del aprendizaje móvil en sus estudios, mientras que el $21 \%$ no realizó aprendizaje móvil en sus actividades, dado que se referían a estudios de análisis de documentos.

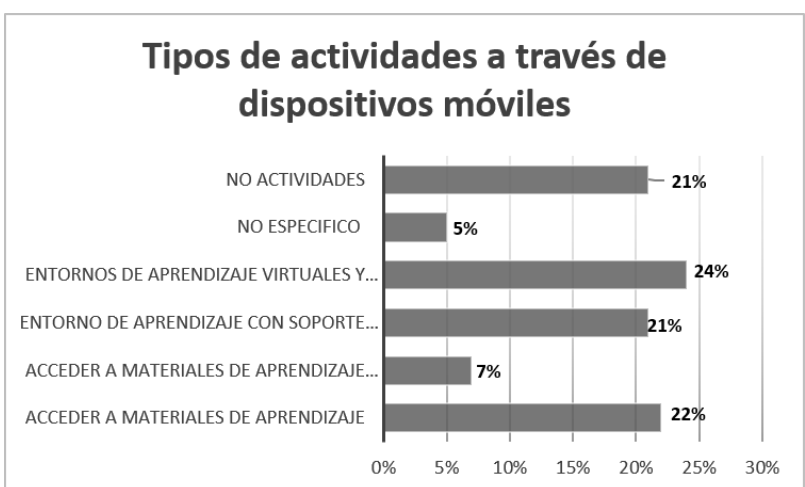

Fig.3. Tipos de actividades a través de dispositivos móviles

Del mismo modo, se realizó una revisión literaria sobre el impacto del programa BYOD (Bring your own device), "trae tu propio dispositivo" en la educación superior [63], en el cual se logra extraer información importante frente a las actividades educativas que realizan los estudiantes a través de los dispositivos móviles. En la Figura 4 se logra apreciar una interacción mayor de los estudiantes por medio de sus móviles en los LMS institucionales (15\%), seguido de la interacción por medio de SMS (13\%), las redes sociales y el poscasting tienen porcentajes similares (12\%), en este orden continúa la mensajería instantánea (11\%), blogging (10\%), Facebook (9\%), microblogging $(7 \%)$, wikis (5\%), códigos QR $(2 \%)$, 
objetos en 3D (2) y por último la realidad aumentada (1\%).



Fig.4. Actividades a través de dispositivos móviles [63]

Al mismo tiempo, teniendo una aproximación hacia un futuro no muy lejado, el aprendizaje móvil no se centrará en app [64], sino en una mayor conectividad para fortalecer y enriquecer las experiencias de aprendizaje de los estudiantes por medio de contenido adaptativo a diferentes dispositivos móviles, sincronización entre estos, y una mayor interactividad por medio del uso de la realidad aumentada, la realidad virtual y la realidad mixta. En adición, se argumenta sobre la tendencia de la utilización de la realidad aumentada centrada en una mayor interacción con los estudiantes donde el aprendizaje se exprese en el contexto físico local complementado por una superposición de audio o video [65].

Otra de las tendencias que se vienen trabajando con más fuerza es la integración de la inteligencia artificial en el aprendizaje móvil. Esta se va abriendo camino en el contexto educativo, los esfuerzos se van centrando en mejorar los sistemas de gestión de la educación, LMS impulsados por intelegencia artificial, aplicaciones educativas orientadas a fortalecer el aprendizaje personalizado, con el fin de apoyar la labor docente y afrontar los desafios de los sistemas educativos en diferente diveles educativos [66]. Igualmente, la inteligencia artificial centrada en aprendizaje móvil que integren ambientes educativos, deben potenciar la interactividad, ofrecer asesoria asistida por medio de fashcards, chats o voz [67]. Es decir, centrado en: sistemas expertos de enseñanza inteligente, motores de recuperación de información, sistemas de aprendizaje inductivo, redes inteligentes de hardware y sistema de apoyo a decisiones [68, 69, 70].
En el caso de las personas con deficiencias congnitivas muestran motivación e interés en la interacción con estos asistentes debido a la posibilidad de simular un conversación con humanos y a disminur la sensación de soledad que estas personas sienten, logrando fortalecer sus procesos de aprendizaje [71].

\section{DISCUSIÓN}

En terminos generales, se espera que el desarrollo del aprendizaje móvil se exprese desde la evaluación de la tecnología y la interacción entre los estudiantes, docentes y contenido. De esta manera, la adopción de políticas como BYOD se incrementará, acompañado de la transformación de contenido adaptativo y con principios de usabilidad en los dispotivos moviles, facilitando el aprendizaje inclusivo para estudiantes con diferentes dificultades fisicas y cognitivas.

El uso de M-Learning es una tendencia a nivel mundial, pese a que en países desarrollados se legitima por parte de los estudiantes, en países en vía de desarrollo las instituciones hacen importantes esfuerzos por estar a la vanguardia y reducir la brecha digital, en diversos niveles como la educación superior [36, 73], primaria y secundaria [74]. Al respecto Colombia a través de programas como "Colombia vive digital" ha masificado el desarrollo de infraestructura orientada a dar conexión a Internet y facilitar el acceso a dispositivos móviles, principalmente tabletas.

Por otro lado, se deben establecer como permanentes algunas estrategias adoptadas frente a la emergencia provocada por pandemia del CODIV-19; en donde el Estado Colombiano permitió el acceso gratuito a Internet de las líneas de telefonía celular prepagadas, con la intencionalidad de garantizar el desarrollo de las actividades escolares. De esta manera, se permitiría el ingreso a contenido digital diverso de estudiantes no solamente de zonas apartadas, que cuentan principalmente con un teléfono celular, contribuyendo a la calidad y cobertura educativa.

\section{CONCLUSIONES Y TRABAJO FUTURO}

Con la revisión sistemática de literatura y los interrogantes establecidos, se evidencia que las investigaciones en el campo del M-Learning se han 
orientado al análisis de las percepciones y el nivel de aceptación de estudiantes y docentes, lo que limita la medición objetiva del impacto en el rendimiento académico y desarrollo de prácticas innovadoras. Es fundamental establecer medidas cuantitativas e instrumentos en el estudio del $M$ Learning para enriquecer el repositorio de experiencia. Aún así, se han identificado esfuerzos para evaluar la usabilidad de sistemas de aprendizaje móvil con diferentes actores evidenciándose un alto porcentaje de aceptación entre ellos [72].

El M-Learning es una estrategia de aprendizaje que conduce a la redefinición del papel docente dentro y fuera del aula de clase, pues el fácil acceso a contenidos brinda espacios para involucrar la academia con el mundo real, experimentar a través de información actualizada y reflexionar con experiencias globales. Por esta razón, se debe iniciar su regulación para crear ecosistemas de aprendizaje sanos y productivos, pues la nomofobia, el cyberbuying, el sexting, distracciones y otro tipo de riesgos, son grandes retos por enfrentar, ya que la prohibición no es la solución factible en un mundo hiperconectado.

La adaptación de plataformas y contenidos educativos orientados al M-Learning deben ser una de las premisas para tener en cuenta, dado que los dispositivos móviles son la base para el desarrollo de otras estrategias. Las futuras investigaciones a este tema ofrecen la oportunidad de ahondar en la propuesta de modelos y parámetros para el desarrollo e implementación de contenidos y plataformas $M$-Learning, donde converjan con otras innovaciones educativas del e-Learning. Adicionalmente, se espera un mayor desarrollo de experiencias inmersivas por medio de la realidad aumentada, realidad virtual, realidad mixta, gamificación, laboratorios virtuales y remotos, además de una mayor integración de la inteligencia artificial que permita fortalecer la labor docente y enfrentar los diferentes desafíos del sistema educativo.

\section{REFERENCIAS BIBLIOGRÁFICAS}

[1] A. Oppenheimer, Crear o morir, Miami: Debate, 2014.

[2] Vera, V. D. G., \& López, C. Q. (2019). Aceptación del M-learning: Un Análisis de
Sentimientos basado en Minería de Texto. Cuaderno Activa, 11, 45-50.

[3] Alam, T., \& Aljohani, M. (2020). M-Learning: Positioning the Academics to the Smart devices in the Connected Future. JOIV : International Journal on Informatics Visualization, 4(2).

[4] Velásquez, J. D. (2015). Una Guía Corta para Escribir Revisiones Sistemáticas de Literatura Parte 3. Dyna, 82(189), 9-12.

[5] Guirao Goris, S. J. A. (2015). Utilidad y tipos de revisión de literatura. Ene, 9(2), 0-0.

[6] P. Ibáñez Carrasco y G. García Torres, Infomática I, vol. I, M. Alegría Estrada, Ed., México D.F: Cengage Learning Editores, S.A., 2009.

[7] Fajardo-Dolci, Germán, \& H, Manuel Ramiro (2015). Innovación. Revista Médica del Instituto Mexicano del Seguro Social, 53(5),532-533.[fecha de Consulta 4 de Mayo de 2020]. ISSN: 0443-5117. Disponible en: https://www.redalyc.org/articulo.oa?id=4577/457744 939001.

[8] Moreno Marchal, J. (2014). Aprender a innovar: una experiencia online. Education In The Knowledge Society (EKS), 15(3), 96-119. doi:10.14201/eks.12220.

[9] Beltrán Meza, M. C. (2017). Innovación en el sector acuícola. Ra Ximhai, 13(3).

[10] Blanco, Á. F. (2014). Editorial "Innovación educativa en la sociedad del conocimiento". Teoría de la Educación. Educación y Cultura en la Sociedad de la Información, 15(3), 1-3.

[11] Parra Robledo, R. (2016). La Innovación educativa contribuye a la inclusión socioeducativa. Didáctica, innovación y multimedia, (34), 0001-13.

[12] Walder, A. M. (2017). Pedagogical Innovation in Canadian higher education: Professors' perspectives on its effects on teaching and learning. Studies in Educational Evaluation, 54, 71-82.

[13] Kostis, P. C., Kafka, K. I., \& Petrakis, P. E. (2018). Cultural change and innovation performance. Journal of Business Research, 88, 306-313. 
[14] Garnica, G. R. (2016). Educación informal en emprendimiento y creatividad en escuelas innovadoras. Opción, 32(12), 425-443.

[15] Keinänen, M., Ursin, J., \& Nissinen, K. (2018). How to measure students' innovation competences in higher education: Evaluation of an assessment tool in authentic learning environments. Studies in Educational Evaluation, 58, 30-36.

[16] Toombs, A. L., Bardzell, S., \& Bardzell, J. (2015, April). The proper care and feeding of hackerspaces: Care ethics and cultures of making. In Proceedings of the 33rd annual ACM conference on human factors in computing systems (pp. 629$638)$.

[17] Liu, Y., Tang, Y., Bi, X., Zhou, X., Zhang, J., \& Wang, J. (2018, May). Cultivation and Practice of College Students' Innovative Entrepreneurship Based on Second Classroom. In Proceedings of the 2018 International Conference on Distance Education and Learning (pp. 113-116).

[18] Gómez Collado, M. E., Contreras Orozco, L., \& Gutiérrez Linares, D. (2016). El impacto de las tecnologías de la información y la comunicación en estudiantes de ciencias sociales: un estudio comparativo de dos universidades públicas. Innovación educativa (México, DF), 16(71), 61-80.

[19] Schefer-Wenzl, S., Miladinovic, I., \& Ensor, A. (2018, October). A Survey of Mobile Learning Approaches for Teaching Internet of Things. In Interactive Mobile Communication, Technologies and Learning (pp. 215-227). Springer, Cham.

[20] Verdún, N. (2016). Educación virtual y sus configuraciones emergentes: Notas acerca del elearning, b-learning y m-learning. Háblame de TIC, 3, 67-88.

[21] Mojarro Aliaño, Á. (2019). Mobile learning en la Educación Superior: una alternativa educativa en entornos interactivos de aprendizaje.

[22] Al-Emran, M., Arpaci, I., \& Salloum, S. A. (2020). An empirical examination of continuous intention to use m-learning: An integrated model. Education and Information Technologies, 1-20.

[23] Hamidi, H., \& Chavoshi, A. (2018). Analysis of the essential factors for the adoption of mobile learning in higher education: A case study of students of the University of Technology. Telematics and Informatics, 35(4), 1053-1070.

[24] Ketel, M. (2014, March). Cloud services for elearning. In Proceedings of the 2014 ACM Southeast Regional Conference (pp. 1-4).

[25] Ruangvanich, S., \& Nilsook, P. (2018, May). Students' Usage of Mobile Learning on Information Technology and Communication Subject for Grade 9 Students. In Proceedings of the 2018 International Conference on Distance Education and Learning (pp. 38-43).

[26] Adly, I., Fadel, M., El-Baz, A., \& Amin, H. (2018, May). Interactive Mobile Learning Platform at the British University in Egypt. In Proceedings of the 7th International Conference on Software and Information Engineering (pp. 97-101).

[27] Hsieh, W. M., \& Tsai, C. C. (2017). Taiwanese high school teachers' conceptions of mobile learning. Computers \& Education, 115, 82-95.

[28] Anshari, M., Almunawar, M. N., Shahrill, M., Wicaksono, D. K., \& Huda, M. (2017). Smartphones usage in the classrooms: Learning aid or interference?. Education and Information Technologies, 22(6), 3063-3079.

[29] Cota, C. X. N., Díaz, A. I. M., \& Duque, M. Á. R. (2014, September). Evaluation framework for mlearning systems: Current situation and proposal. In Proceedings of the XV International Conference on Human Computer Interaction (pp. 1-3).

[30] Valderrama Bahamóndez, E. D. C., Pfleging, B., Henze, N., \& Schmidt, A. (2014, September). A long-term field study on the adoption of smartphones by children in panama. In Proceedings of the 16th international conference on Humancomputer interaction with mobile devices \& services (pp. 163-172).

[31] Choi, G. W., Land, S. M., \& Zimmerman, H. T. (2018). Investigating children's deep learning of the tree life cycle using mobile technologies. Computers in Human Behavior, 87, 470-479.

[32] Fabian, K., \& MacLean, D. (2014). Keep taking the tablets? Assessing the use of tablet devices in learning and teaching activities in the Further Education sector. Research in Learning Technology, 22. 
[33] Park, E., Han, J., Kim, K. J., Cho, Y., \& del Pobil, A. P. (2018, January). Effects of Screen Size in Mobile Learning Over Time. In Proceedings of the 12th International Conference on Ubiquitous Information Management and Communication (pp. 1-5).

[34] Tomaschko, M., \& Hohenwarter, M. (2017, December). Integrating mobile and sensory technologies in mathematics education. In Proceedings of the 15th International Conference on Advances in Mobile Computing \& Multimedia (pp. 39-48).

[35] Cahya, R. N., Suprapto, E., \& Lusiana, R. Development of Mobile Learning Media Based Android to Suport Students Understanding.

[36] Al-Emran, M., Mezhuyev, V., \& Kamaludin, A. (2020). Towards a conceptual model for examining the impact of knowledge management factors on mobile learning acceptance. Technology in Society, 101247.

[37] Oliveira, D. T., Carvalho, M. D., \& Paixão, T. L. S. (2016, November). Ubiquitous Computing: Gestures Interaction Applied the Learning Disabilities in Process Literacy. In Proceedings of the 22nd Brazilian Symposium on Multimedia and the Web (pp. 107-110).

[38] Aktekin, N. Ç., Çelebi, H., \& Aktekin, M. (2018). Let's Kahoot! Anatomy. International Journal of Morphology, 36(2).

[39] Furió, D., Juan, M. C., Seguí, I., \& Vivó, R. (2015). Mobile learning vs. traditional classroom lessons: a comparative study. Journal of Computer Assisted Learning, 31(3), 189-201.

[40] Remmer, M., Denami, M., \& Marquet, P. (2017, March). Why Pokémon GO is the future of school education: effects of $A R$ on intrinsic motivation of children at elementary school. In Proceedings of the Virtual Reality International Conference-Laval Virtual 2017 (pp. 1-5).

[41] Zuckerman, O., Gal-Oz, A., Peretz, O., Weisberg, O., \& Tarrasch, R. (2015, August). Leveraging Mobile Technology to Engage College Students in Scientific Research. In Proceedings of the 17th International Conference on HumanComputer Interaction with Mobile Devices and Services (pp. 470-477).
[42] Palomo-Duarte, M., Berns, A., Dodero, J. M., \& Cejas, A. (2014, October). Foreign language learning using a gamificated APP to support peerassessment. In Proceedings of the Second International Conference on Technological Ecosystems for Enhancing Multiculturality (pp. 381386).

[43] Xiao, X., Pham, P., \& Wang, J. (2015, November). AttentiveLearner: adaptive mobile MOOC learning via implicit cognitive states inference. In Proceedings of the 2015 ACM on International Conference on Multimodal Interaction (pp. 373-374).

[44] Karoui, A., Marfisi-Schottman, I., \& George, S. (2017, October). A Nested Design Approach for Mobile Learning Games. In Proceedings of the 16th World Conference on Mobile and Contextual Learning (pp. 1-4).

[45] Briz-Ponce, L., Juanes-Méndez, J. A., GarcíaPeñalvo, F. J., \& Pereira, A. (2016). Effects of mobile learning in medical education: a counterfactual evaluation. Journal of medical systems, 40(6), 136.

[46] Reis, S., \& Almeida, A. M. (2016, December). The use of mobile devices to support daily routines of teens with Down syndrome: a case study. In Proceedings of the 7th International Conference on Software Development and Technologies for Enhancing Accessibility and Fighting Info-exclusion (pp. 154-161).

[47] Ozdamli, F., \& Uzunboylu, H. (2015). M-learning adequacy and perceptions of students and teachers in secondary schools. British Journal of Educational Technology, 46(1), 159-172.

[48] Hashim, K. F., Tan, F. B., \& Rashid, A. (2015). Adult learners' intention to adopt mobile learning: A motivational perspective. British Journal of Educational Technology, 46(2), 381-390.

[49] Sobaih, A. E. E., Moustafa, M. A., Ghandforoush, P., \& Khan, M. (2016). To use or not to use? Social media in higher education in developing countries. Computers in Human Behavior, 58, 296-305.

[50] Castro, G. G., Dominguez, E. L., Velazquez, Y. H., Matla, M. Y. R., Toledo, C. B. E., \& Hernandez, 
S. E. P. (2016). MobiLearn: context-aware mobile learning system. IEEE Latin America Transactions, 14(2), 958-964.

[51] Brown, T. H., \& Mbati, L. S. (2015). Mobile learning: Moving past the myths and embracing the opportunities. International Review of Research in Open and Distributed Learning, 16(2), 115-135.

[52] Tan, E., \& So, H. J. (2014). Rethinking the impact of activity design on a mobile learning trail: The missing dimension of the physical affordances. IEEE Transactions on Learning Technologies, 8(1), 98-110.

[53] Alter, A. (2018). Irresistible. Superfunds Magazine, (440), 58.

[54] Mendoza, J. S., Pody, B. C., Lee, S., Kim, M., \& McDonough, I. M. (2018). The effect of cellphones on attention and learning: The influences of time, distraction, and nomophobia. Computers in Human Behavior, 86, 52-60.

[55] O'Bannon, B. W., \& Thomas, K. M. (2015). Mobile phones in the classroom: Preservice teachers answer the call. Computers \& Education, 85, 110-122.

[56] Burns, M. (2015). Not Just for Students: Mobile teacher professional development. eLearn, 2015(March).

[57] Dávila, M. R. M. (2020). M-Learning: características, ventajas y desventajas, uso. Revista Tecnológica-Educativa Docentes 2.0, 8(1), 50-52.

[58] Ramírez, L. L., \& Montalvo, J. A. C. (2019). El aprendizaje móvil en la educación superior, frontera del conocimiento: 2014-2018. Revista Electrónica de Investigación e Innovación Educativa-REIIE ISSN: 2448-556X, 4(3), 27-41.

[59] Delgado, R. Z. (2019). El M-Learning, las ventajas de la utilización de dispositivos móviles en el proceso autónomo de aprendizaje. ReHuSo: Revista de Ciencias Humanísticas y Sociales, 4(3), 29-38.

[60] Marco, F. A., Penichet, V. M., \& Gallud, J. A. (2015, August). What happens when students go offline in mobile devices?. In Proceedings of the 17th International Conference on Human-Computer
Interaction with Mobile Devices and Services Adjunct (pp. 1199-1206).

[61] Shawar, B. A. (2017, September). Neither Completely M-Nor E-Learning: Integrating both is the Solution. In Proceedings of the 2017 International Conference on Cloud and Big Data Computing (pp. 112-118).

[62] Lai, C. L. (2019). Trends of mobile learning: A review of the top 100 highly cited papers. British Journal of Educational Technology.

[63] Sundgren, M. (2017). Blurring time and place in higher education with bring your own device applications: a literature review. Education and Information Technologies, 22(6), 3081-3119.

[64] Alexander, B., Ashford-Rowe, K., BarajasMurph, N., Dobbin, G., Knott, J., McCormack, M., ... \& Weber, N. (2019). EDUCAUSE Horizon Report 2019 Higher Education Edition (pp. 3-41). EDU19.

[65] Hwang, G. J., \& Fu, Q. K. (2020). Advancement and research trends of smart learning environments in the mobile era. International Journal of Mobile Learning and Organisation, 14(1), 114-129.

[66] UNESCO - Mobile Learning Week 2019: Deciphering the impact of Artificial Intelligence on education. Disponible en: https://en.unesco.org/news/mobile-learning-week2019-deciphering-impact-artificial-intelligenceeducation [consultado el 28 de mayo de 2020].

[67] Marwan, A. (2019). Impact of artificial intelligence on education for employment:(learning and employability Framework).

[68] Khan, M. A., Tripathi, A., Dixit, A., \& Dixit, M. (2019, January). Correlative Analysis and Impact of Intelligent Virtual Assistants on Machine Learning. In 2019 11th International Conference on Computational Intelligence and Communication Networks (CICN) (pp. 133-139). IEEE.

[69] Polyakov, E. V., Mazhanov, M. S., Rolich, A. Y., Voskov, L. S., Kachalova, M. V., \& Polyakov, S. V. (2018, March). Investigation and development of the intelligent voice assistant for the Internet of Things using machine learning. In 2018 Moscow Workshop on Electronic and Networking Technologies (MWENT) (pp. 1-5). IEEE. 
[70] Abdolrahmani, A., Kuber, R., \& Branham, S. M. (2018, October). " Siri Talks at You" An Empirical Investigation of Voice-Activated Personal Assistant (VAPA) Usage by Individuals Who Are Blind. In Proceedings of the 20th International ACM SIGACCESS Conference on Computers and Accessibility (pp. 249-258).

[71] Terzopoulos, G., \& Satratzemi, M. (2019, September). Voice Assistants and Artificial Intelligence in Education. In Proceedings of the 9th Balkan Conference on Informatics (pp. 1-6).

[72] Pensabe-Rodriguez, A., Lopez-Dominguez, E., Hernandez-Velazquez, Y., Dominguez-Isidro, S., \& de la Calleja, J. (2020). Context-Aware Mobile Learning System: Usability Assessment Based on a Field Study. Telematics and Informatics, 101346.

[73] Okai-Ugbaje, S., Ardzejewska, K., \& Imran, A. (2020). Readiness, Roles, and Responsibilities of Stakeholders for Sustainable Mobile Learning Adoption in Higher Education. Education Sciences, 10(3), 49.

[74] López-Faican, L., \& Jaen, J. (2020). Emofindar: Evaluation of a mobile multiplayer augmented reality game for primary school children. Computers \& Education, 149, 103814. 